\title{
Advances in medical education and practice: student perceptions of the flipped classroom
}

This article was published in the following Dove Press journal:

Advances in Medical Education and Practice

13 January 2017

Number of times this article has been viewed

\section{Christopher J Ramnanan' Lynley D Pound ${ }^{2}$}

'Department of Innovation in Medical Education, Faculty of Medicine, University of Ottawa, ${ }^{2}$ Ottawa Hospital Research Institute, Ottawa, ON, Canada
Correspondence: Christopher J Ramnanan

Department of Innovation in Medical Education, Faculty of Medicine, University of Ottawa, 45I Smyth Road, Ottawa, ON KIH 8M5, Canada

Tel +l 6135625800 ext 8702

Email cramnana@uottawa.ca

\begin{abstract}
The flipped classroom (FC) approach to teaching has been increasingly employed in undergraduate medical education in recent years. In FC applications, students are first exposed to content via online resources. Subsequent face-to-face class time can then be devoted to student-centered activities that promote active learning. Although the FC has been well received by students in other contexts, the perceptions of medical students regarding this innovation are unclear. This review serves as an early exploration into medical student perceptions of benefits and limitations of the FC. Medical students have generally expressed strong appreciation for the pre-class preparation activities (especially when facilitated by concise, readily accessed online tools) as well as for interactive, engaging small group classroom activities. Some students have expressed concerns with the FC and noted that suboptimal student preparation and insufficient direction and structure during active learning sessions may limit the student-centered benefits. Although students generally perceive that FC approaches can improve their learning and knowledge, this has not been conclusively shown via performances on assessment tools, which may be related to caveats with the assessment tools used. In any case, lifelong self-directed learning skills are perceived by medical students to be enhanced by the FC. In conclusion, medical students have generally expressed strong satisfaction with early applications of the FC to undergraduate medical education, and generally prefer this method to lecture-based instruction.
\end{abstract}

Keywords: flipped classroom, active learning, student perceptions, undergraduate medical education, medical student, case-based learning, problem-based learning, team-based learning

\section{Introduction}

The traditional classroom lecture is still commonly used in undergraduate medical education. However, classroom lectures can be considered teacher-centered strategies that are conducive to passive learning on behalf of learners. ${ }^{1}$ Conversely, active learning strategies (ie, opportunities for students to participate, self-direct, and engage in their own learning) are not only supported by adult learning theories but have also been shown to be beneficial to long-term learning and the development of selfdirected learning skills. ${ }^{2,3}$ Moreover, accreditation standards now formally demand that medical programs include opportunities for medical students to work in active learning environments, so that they can develop lifelong learning skills (independent identification, appraisal, analysis, and synthesis of knowledge) desirable of modern physicians. ${ }^{4}$ Finally, there is now increased emphasis in formal medical curricula on developing competencies related to professionalism, medical ethics, the humanities, interprofessional collaboration, and other areas, resulting in less curricular face-to-face time devoted to many of the basic and clinical sciences. ${ }^{4}$ 
Given these temporal, accreditation, and pedagogical considerations in undergraduate medical education, many medical educators are now applying elements of the flipped classroom (FC) educational strategy to their teaching. In the conventional classroom model, students are first exposed to educational content via professor-centered, didactic lectures. Learning may then be reinforced by postlecture assignments (homework) that offer opportunities for students to apply their newly gained knowledge independently (typically without interaction or contact with classmates or teachers). In the FC model, learners are first exposed to educational content prior to formal class sessions via readings, videos, or other electronic exercises that have been formally assigned. Given that students have already acquired knowledge through this initial "homework" phase, the subsequent classroom time can then be dedicated to activities that allow students to apply their knowledge to challenging problems in a setting that promotes collaboration with peers and feedback and direction from teachers. In terms of Bloom's revised taxonomy, the traditional lecture has largely invested classroom time (where teachers and students interact faceto-face) in promoting lower level cognitive work (gain and comprehension of factual knowledge), whereas the FC offers the opportunity for students to engage in higher order cognition (application, analysis, evaluation, and synthesis of knowledge). ${ }^{5}$

Although faculty enthusiasm, positive student perceptions, and gains in learning outcomes have been, to some degree, characterized in various disciplines, ${ }^{6-9}$ it remains unclear how the $\mathrm{FC}$ is received by undergraduate medical students. The goal of the present scoping review is therefore to explore the application of FC methodology in undergraduate medical education. This review will aim to identify trends in both the pre-class and in-class phases of the approach. In addition, this review will focus on the medical student perspective and, specifically, define medical student perceptions (strengths as well as caveats) of this method as well as the impact (perceived and actual) that the FC has on medical student learning.

\section{Methods}

We performed a review of the literature, using the established scoping review framework delineated by Arksey and O'Malley, ${ }^{10}$ to explore the literature defining applications of the FC in undergraduate medical education. Specifically, literature was searched, studies were collated, and data synthesized in this scoping review with the goal of addressing the following questions:
1. What subject matter has been delivered using the FC approach (for preclinical and for clinical year medical students)?

2. Which tools and teaching methods have been used for pre-class and during-class (active learning) phases in medical school?

3. How do medical students perceive (both strengths and caveats) the $\mathrm{FC}$ approach?

4. What is the impact of the FC on medical student learning?

To identify studies that were relevant to this review, the authors first defined the FC method. Although various descriptions outside the context of medical education exist, ${ }^{6-9}$ the core principles that appear most consistently across applications of the FC can be simplified to include: 1) the assignment of preparatory work (primarily viewing of videos or utilizing other audiovisual and electronic learning [e-learning] resources that can be accessed asynchronously, repeatedly, and on demand) prior to classroom sessions designed to introduce the relevant content to students and 2) active learning classroom activities that are dedicated to the application of knowledge in the context of feedback from teachers (Table 1).

As such, the authors searched Scopus, Web of Science, and PubMed databases in September 2016 for articles meeting the following inclusion criteria:

1. The learners in the article's "FC" application were undergraduate medical students (both preclinical and clinical applications were included);

2. The article detailed the application of the FC methodology to a sufficient degree to fit the descriptions above;

3. The article presented some degree of original data related to the evaluation of their FC approach, specifically defining student perceptions of the application and/or impact on learning outcomes;

4. Articles were chosen, which were published during or after 2012 (2012 to September 2016), as 2012 was the year of the first publication of an application of the FC to medical student teaching; $;{ }^{11}$ and

5. Full texts of the articles were accessible in English.

The abstracts of all articles derived from initial searches were read, and articles that fit the selection criteria above were selected (by CJR and LDP) to be read in full. The reference lists of review articles (not containing original data evaluating the FC methodology, and therefore themselves were not included) were searched for relevant articles fitting the inclusion criteria above (the abstracts were read for initial screening, as before by CJR and LDP). At this point, the list of articles still eligible for inclusion (after analysis of 
Table I Characteristics of the traditional classroom, the FC, and the undergraduate medical education FC based on studies from this review

\begin{tabular}{|c|c|c|}
\hline & Phase I - content orientation & Phase 2 - application of knowledge \\
\hline \multicolumn{3}{|l|}{ Traditional classroom } \\
\hline $\begin{array}{l}\text { Students: passive learners } \\
\text { Teachers: Sage on the Stage }\end{array}$ & $\begin{array}{l}\text { Classrooms are dedicated to } \\
\text { lecture-based teacher-centered activities }\end{array}$ & $\begin{array}{l}\text { Students may apply knowledge from prior lectures to } \\
\text { "homework assignments" (independent application, } \\
\text { outside the context of peer or faculty interaction) }\end{array}$ \\
\hline \multicolumn{3}{|l|}{ Flipped classroom } \\
\hline $\begin{array}{l}\text { Students: active learners } \\
\text { Teachers: Guide on the Side, facilitating } \\
\text { student inquiry }\end{array}$ & $\begin{array}{l}\text { Through "homework", students are } \\
\text { introduced to basic content via online } \\
\text { resources }\end{array}$ & $\begin{array}{l}\text { Classrooms can now be dedicated to creating active } \\
\text { learning environments that foster deeper learning. } \\
\text { Students may tackle more challenging concepts in the } \\
\text { context of working with their peers and with access } \\
\text { to faculty }\end{array}$ \\
\hline
\end{tabular}

Undergraduate medical education FC

Elements utilized in medical education FCs from reviewed studies
Lecture videos, podcasts, formative quizzes, $\quad C B L, T B L, P B L$, small group discussions e-learning modules

Abbreviations: FC, flipped classroom; CBL, case-based learning; TBL, team-based learning; PBL, problem-based learning.

abstracts) were read in full by both authors, and those chosen to be included for review were discussed, agreed upon, and reviewed by both authors.

\section{Results}

The initial search strategies yielded 123 original candidate articles, but after abstract review, 43 articles remained eligible for inclusion (Figure 1). Among the common reasons for article exclusion were the following: the learners described were not medical students, no data regarding the evaluation of their application were provided, and sufficient information to allow characterization of the classroom portion of the application as active learning was not provided. After reading full texts, discussion, and agreement by both authors, 17 additional studies were excluded for reasons including lack of primary data, nonmedical student learners, and insufficient program evaluation data or details regarding the pre-class and/or during-class phases of the flipped interventions (Figure 1). As a result, 26 articles were selected for inclusion in this review.

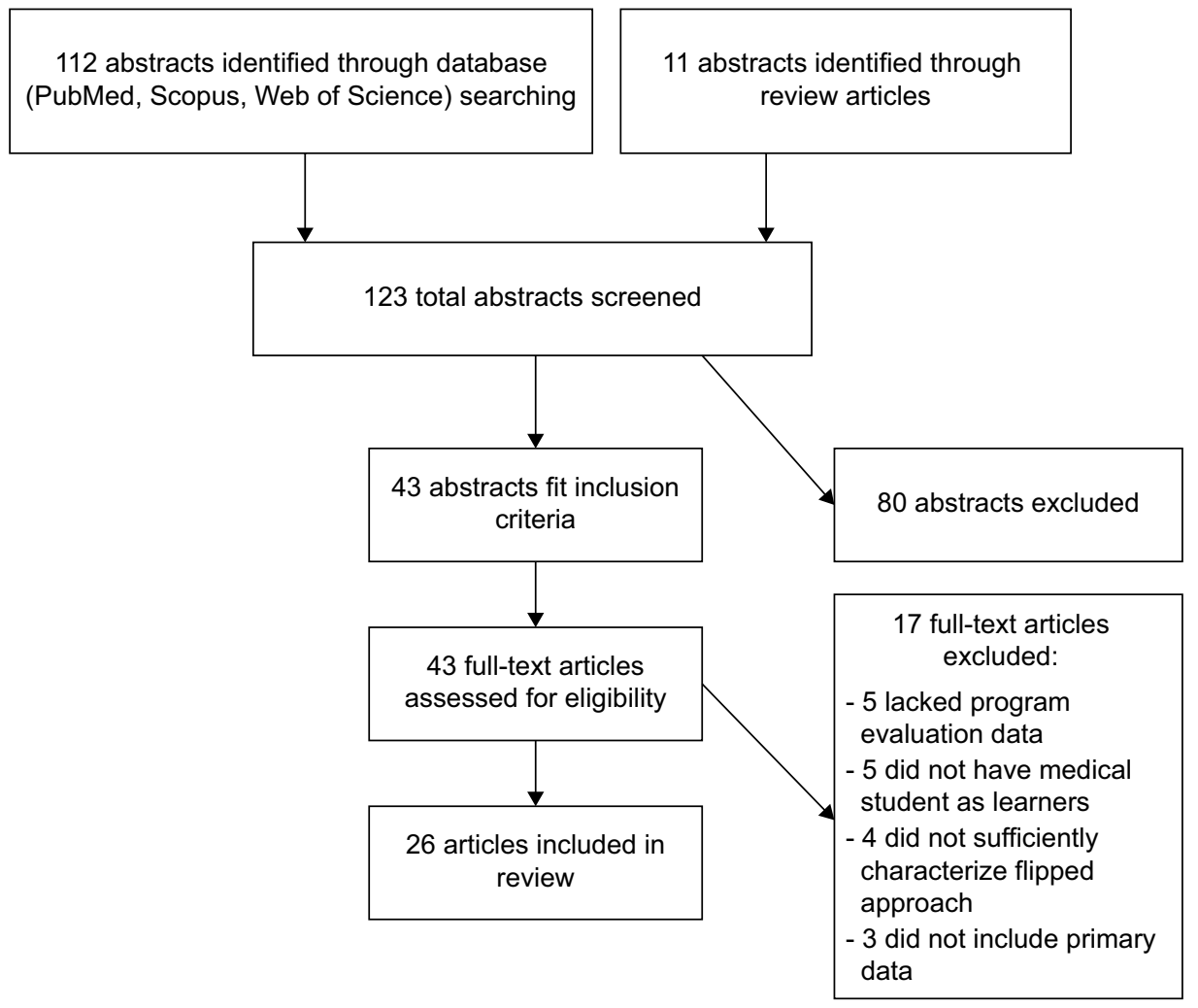

Figure I PRISMA style schematic for our scoping review. 


\section{Subject matter in preclinical and clinical FC approaches}

The FC methodology has been applied in various contexts in both preclinical and clinical educational settings with medical student learners. Preclinical application of FC elements was concentrated on the basic sciences (Table 2), such as biochemistry ${ }^{11,12}$ and the anatomical sciences. ${ }^{13-15}$ The incorporation of FC pedagogy in medical anatomy classes has been suggested to be driven, at least in part, by markedly reduced curricular time afforded to the teaching of the subject. ${ }^{15}$ Other preclinical FC applications included education related to the humanities, ${ }^{16}$ epidemiology, ${ }^{17}$ rheumatology, ${ }^{18}$ hematology ${ }_{1}^{19}$ and point-of-care ultrasound (POCUS) ${ }^{20}$ Similar to the case with anatomy, these subjects are believed to be important to the education of modern physicians, but curricular leadership may have difficulty finding formal curricular time to devote to these topics. As such, FC methodology may be ideal not only for introducing active learning to the in-class sessions related to these disciplines but also for introducing students to valuable concepts outside of curricular time.

FC methodology at the clinical level of medical school has, to date, been applied to various initiatives that are related to core components of undergraduate medical education (Table 3), including emergency medicine, ${ }^{21,22}$ evidencebased medicine (EBM), ${ }^{23,24}$ obstetrics and gynecology, ${ }^{25-27}$ surgery, ${ }^{28,29}$ differential diagnosis, ${ }^{30}$ and radiology. ${ }^{31,32}$ In addition, the inverted classroom methodology has been applied to topics such as geriatric medicine, ${ }^{33}$ disaster medicine, ${ }^{34}$ advanced cardiac life support,,$^{35}$ and an elective related to medicine as a business. ${ }^{36}$ Generally, authors did not allude

Table 2 Studies that characterized medical student perceptions to FC approaches in preclinical subjects

\begin{tabular}{|c|c|c|c|c|c|}
\hline $\begin{array}{l}\text { Study authors, } \\
\text { year }\end{array}$ & $\begin{array}{l}\text { Subject matter } \\
\text { (learner level, if } \\
\text { described) }\end{array}$ & $\begin{array}{l}\text { Primary pre- } \\
\text { session online } \\
\text { resources }\end{array}$ & $\begin{array}{l}\text { In-session active } \\
\text { learning activities }\end{array}$ & $\begin{array}{l}\text { Comparison } \\
\text { group }\end{array}$ & $\begin{array}{l}\text { Main outcomes from flipped } \\
\text { elements }\end{array}$ \\
\hline $\begin{array}{l}\text { Prober and } \\
\text { Heath," } 2012\end{array}$ & Biochemistry (MI) & Video lectures & $\begin{array}{l}\text { Discussions of clinical } \\
\text { vignettes }\end{array}$ & $\begin{array}{l}\text { Previous cohort } \\
\text { (LB) }\end{array}$ & $\begin{array}{l}\text { Positive effect on student perceptions } \\
\text { and attendance }\end{array}$ \\
\hline $\begin{array}{l}\text { de Fatima } \\
\text { Wardenski } \\
\text { et al, }{ }^{12} 2012\end{array}$ & Biochemistry (MI) & $\begin{array}{l}\text { Lecture slides, } \\
\text { audiovisual resources, } \\
\text { bio-informatics tools, } \\
\text { scientific papers }\end{array}$ & $\begin{array}{l}\text { Forums for small } \\
\text { group work }\end{array}$ & None & $\begin{array}{l}\text { Students perceived benefits related } \\
\text { to knowledge and motivation to } \\
\text { learn and need for greater support/ } \\
\text { direction for both pre-class and in- } \\
\text { class activities }\end{array}$ \\
\hline $\begin{array}{l}\text { Veeramani } \\
\text { et al, }{ }^{13} 2015\end{array}$ & $\begin{array}{l}\text { Clinical } \\
\text { neuroanatomy (MI) }\end{array}$ & $\begin{array}{l}\text { Web-based modules } \\
\text { and text-based } \\
\text { resources }\end{array}$ & $\begin{array}{l}\text { Clinical case-based } \\
\text { discussions }\end{array}$ & $\begin{array}{l}\text { Previous cohort } \\
\text { (LB) }\end{array}$ & $\begin{array}{l}\text { Performance on examination } \\
\text { improved; students perceived increased } \\
\text { engagement and stated preference for } \\
\text { FC relative to didactic lectures }\end{array}$ \\
\hline $\begin{array}{l}\text { Morton and } \\
\text { Colbert-Getz, }{ }^{14} \\
2016\end{array}$ & Anatomy (MI) & $\begin{array}{l}\text { Video lectures, } \\
\text { workbook } \\
\text { assignments }\end{array}$ & $\begin{array}{l}\text { Large group discussion } \\
\text { (think, pair, share) } \\
\text { approach to problem } \\
\text { sets }\end{array}$ & $\begin{array}{l}\text { Previous cohort } \\
\text { (LB) }\end{array}$ & $\begin{array}{l}\text { No difference on knowledge-based } \\
\text { items, but increased performance on } \\
\text { items that assessed higher levels of } \\
\text { cognition }\end{array}$ \\
\hline $\begin{array}{l}\text { Whelan et al, }{ }^{15} \\
2016\end{array}$ & $\begin{array}{l}\text { Anatomy (MI and } \\
M 2)\end{array}$ & $\begin{array}{l}\text { Audiovisual } \\
\text { resources, lecture } \\
\text { slides }\end{array}$ & $\begin{array}{l}\text { Modified TBL } \\
\text { (student-driven } \\
\text { approach to small } \\
\text { group learning) }\end{array}$ & $\begin{array}{l}\text { Faculty-driven } \\
\text { approach to small } \\
\text { group learning }\end{array}$ & $\begin{array}{l}\text { Appreciation for benefits of student- } \\
\text { centered learning, but active learning } \\
\text { environment was highly variable } \\
\text { between groups }\end{array}$ \\
\hline $\begin{array}{l}\text { Grossman } \\
\text { et al, }{ }^{16} 2015\end{array}$ & $\begin{array}{l}\text { Humanities, narrative } \\
\text { medicine }\end{array}$ & Multimodal modules & $\begin{array}{l}\text { Interactive activities } \\
\text { (eg, construct } \\
\text { narratives based on } \\
\text { online information) }\end{array}$ & None & $\begin{array}{l}\text { Positive student perceptions regarding } \\
\text { engaging learning environment }\end{array}$ \\
\hline $\begin{array}{l}\text { Evans et al, }{ }^{17} \\
2016\end{array}$ & Epidemiology (MI) & $\begin{array}{l}\text { Lecture videos, texts, } \\
\text { and lecture slides }\end{array}$ & $\begin{array}{l}\text { Case-based small } \\
\text { group discussions }\end{array}$ & $\begin{array}{l}\text { Previous cohort } \\
\text { (LB) }\end{array}$ & $\begin{array}{l}\text { Increased student satisfaction with } \\
\text { small group activities, but no impact } \\
\text { on examination performance }\end{array}$ \\
\hline $\begin{array}{l}\text { Sharma et al, }{ }^{18} \\
2015\end{array}$ & Rheumatology & Lecture videos & $\begin{array}{l}\text { Hybrid approach } \\
\text { incorporating } T B L \text { and } \\
C B L\end{array}$ & None & $\begin{array}{l}\text { Positive student feedback (vaguely } \\
\text { described) }\end{array}$ \\
\hline $\begin{array}{l}\text { Sajid et al, }{ }^{19} \\
2016\end{array}$ & Hematology (M3) & Lecture videos & $\begin{array}{l}\text { Application of } \\
\text { knowledge exercises }\end{array}$ & $\begin{array}{l}\text { Previous cohorts } \\
\text { (LB) }\end{array}$ & $\begin{array}{l}\text { High levels of satisfaction; no impact } \\
\text { on examination performance }\end{array}$ \\
\hline $\begin{array}{l}\text { Nelson et al, }{ }^{20} \\
2016\end{array}$ & POCUS (MI) & Lecture videos & $\begin{array}{l}\text { Interactive, hands-on } \\
\text { session using POCUS }\end{array}$ & None & $\begin{array}{l}\text { Both students and faculty expressed } \\
\text { value for initiative }\end{array}$ \\
\hline
\end{tabular}

Abbreviations: FC, flipped classroom; TBL, team-based learning; CBL, case-based learning; MI, first-year medical students; M2, second-year medical students; M3, thirdyear medical students; POCUS, point-of-care ultrasound; LB, lecture-based or teacher-centered approach. 
Table 3 Studies that characterized medical student perceptions to FC approaches in clinical education

\begin{tabular}{|c|c|c|c|c|c|}
\hline $\begin{array}{l}\text { Study } \\
\text { authors, } \\
\text { year }\end{array}$ & $\begin{array}{l}\text { Subject matter } \\
\text { (learner level, if } \\
\text { described) }\end{array}$ & $\begin{array}{l}\text { Primary pre- } \\
\text { session online } \\
\text { resources }\end{array}$ & $\begin{array}{l}\text { In-session active } \\
\text { learning activities }\end{array}$ & $\begin{array}{l}\text { Comparison } \\
\text { group }\end{array}$ & Main outcomes from flipped elements \\
\hline $\begin{array}{l}\text { Patwari and } \\
\mathrm{Yiu}^{21} 2014\end{array}$ & $\begin{array}{l}\text { Emergency medicine } \\
\text { (M3) }\end{array}$ & Lecture videos & $\begin{array}{l}\text { Active learning on } \\
\text { wards }\end{array}$ & None & $\begin{array}{l}\text { Positive overall reception toward online resources, } \\
\text { with some caveats noted }\end{array}$ \\
\hline Lew, ${ }^{22} 2016$ & $\begin{array}{l}\text { Emergency medicine } \\
\text { clerkship (M3 and } \\
\text { M4) }\end{array}$ & Lecture videos & $\begin{array}{l}\text { Case-based small } \\
\text { group discussion }\end{array}$ & None & $\begin{array}{l}\text { Student surveys report preference for flipped approach } \\
\text { vs lectures; facilitators reported perceived high levels } \\
\text { of student engagement }\end{array}$ \\
\hline $\begin{array}{l}\text { Ilic et al, }{ }^{23} \\
2013\end{array}$ & EBM (M2) & $\begin{array}{l}\text { Self-directed } \\
\text { learning tools } \\
\text { (including lecture } \\
\text { videos) }\end{array}$ & $\begin{array}{l}\text { Facilitated } \\
\text { workshop (with } \\
\text { journal club } \\
\text { elements) }\end{array}$ & $\begin{array}{l}\text { Control group } \\
\text { (LB) }\end{array}$ & $\begin{array}{l}\text { Berlin tool (quantitative objective assessment of } \\
\text { EBM competency) did not indicate an effect, though } \\
\text { self-perceived skills in specific areas were improved; } \\
\text { qualitative data from focus groups indicated benefits } \\
\text { (self-directed learning) and limitations (lack of student } \\
\text { preparedness) }\end{array}$ \\
\hline $\begin{array}{l}\text { llic et al, }{ }^{24} \\
2015\end{array}$ & EBM (M3) & $\begin{array}{l}\text { Online self- } \\
\text { directed learning } \\
\text { tools (including } \\
\text { lecture videos) }\end{array}$ & $\begin{array}{l}\text { Application of } \\
\text { knowledge activities } \\
\text { on wards }\end{array}$ & $\begin{array}{l}\text { Randomized } \\
\text { control group }\end{array}$ & $\begin{array}{l}\text { No impact on learning or skills; positive effect on } \\
\text { attitudes toward EBM (surveys) and the use of EBM in } \\
\text { clinic (self-reported) }\end{array}$ \\
\hline $\begin{array}{l}\text { Gillespie, }{ }^{25} \\
2016\end{array}$ & $\begin{array}{l}\text { Obstetrics and } \\
\text { gynecology clerkship } \\
\text { (M3 and M4) }\end{array}$ & $\begin{array}{l}\text { Lecture videos } \\
\text { (narrated } \\
\text { presentations) }\end{array}$ & PBL sessions & $\begin{array}{l}\text { Previous } \\
\text { cohort (LB) }\end{array}$ & $\begin{array}{l}\text { Flipped approach led to increased performance on } \\
\text { various assessments (exams and OSCEs), but led to } \\
\text { reduced scores on gynecology-related items on exams }\end{array}$ \\
\hline $\begin{array}{l}\text { Morgan } \\
\text { et al, }{ }^{26} 2014\end{array}$ & $\begin{array}{l}\text { Obstetrics- } \\
\text { gynecology (M4) }\end{array}$ & $\begin{array}{l}\text { Videos and } \\
\text { readings }\end{array}$ & CBL sessions & $\begin{array}{l}\text { Didactic } \\
\text { components of } \\
\text { same course }\end{array}$ & $\begin{array}{l}\text { Students preferred flipped elements over didactic } \\
\text { elements; knowledge increased (pretest vs posttest) }\end{array}$ \\
\hline $\begin{array}{l}\text { Morgan } \\
\text { et al, }{ }^{27} 2015\end{array}$ & $\begin{array}{l}\text { FC approach } \\
\text { to obstetrics- } \\
\text { gynecology (M4) }\end{array}$ & $\begin{array}{l}\text { Videos, narrated } \\
\text { presentations }\end{array}$ & CBL sessions & $\begin{array}{l}\text { Previous } \\
\text { cohorts (LB) }\end{array}$ & $\begin{array}{l}\text { Flipped elements were positively received (student } \\
\text { surveys); no evidence of knowledge gain (NBME } \\
\text { results) }\end{array}$ \\
\hline $\begin{array}{l}\text { Liebert } \\
\text { et al, }{ }^{28} 2016\end{array}$ & Surgery clerkship & Video lectures & $\begin{array}{l}\text { Review of pretest, } \\
\text { case-based clinical } \\
\text { reasoning }\end{array}$ & $\begin{array}{l}\text { Previous } \\
\text { cohorts (LB) }\end{array}$ & $\begin{array}{l}\text { Self-reported increases in interest in surgical careers; } \\
\text { no difference in NBME results }\end{array}$ \\
\hline $\begin{array}{l}\text { Liebert } \\
\text { et } \mathrm{al}^{129} 2016\end{array}$ & $\begin{array}{l}\text { Simulation-based } \\
\text { surgery clerkship }\end{array}$ & Video lectures & $\begin{array}{l}\text { Review of pretest, } \\
\text { case-based clinical } \\
\text { reasoning }\end{array}$ & None & $\begin{array}{l}\text { Perceived benefits related to self-directed learning, } \\
\text { student accountability }\end{array}$ \\
\hline $\begin{array}{l}\text { Bosner } \\
\text { et al, },^{30} 2015\end{array}$ & $\begin{array}{l}\text { Primary care } \\
\text { diagnosis skills (M4 } \\
\text { and M5) }\end{array}$ & $\begin{array}{l}\text { Video and } \\
\text { audio-recorded } \\
\text { lectures }\end{array}$ & $\begin{array}{l}\text { Interactive small } \\
\text { group laboratory } \\
\text { exercises }\end{array}$ & None & $\begin{array}{l}\text { Positive student perceptions toward interactive small } \\
\text { group approach; interest and engagement were also } \\
\text { enhanced per student surveys and focus groups; } \\
\text { knowledge gained (pretest vs posttest) }\end{array}$ \\
\hline $\begin{array}{l}\text { Belfi et al, }{ }^{31} \\
2015\end{array}$ & $\begin{array}{l}\text { Radiology clerkship } \\
\text { (M3) }\end{array}$ & $\begin{array}{l}\text { Interactive } \\
\text { modules, games, } \\
\text { and simulator }\end{array}$ & $\begin{array}{l}\text { Modified } \\
\text { approaches to } \mathrm{CBL} \\
\text { and } \mathrm{TBL}\end{array}$ & $\begin{array}{l}\text { Didactic } \\
\text { components of } \\
\text { same course }\end{array}$ & $\begin{array}{l}\text { Student surveys characterized positive perceptions } \\
\text { to flipped elements relative to didactic elements; } \\
\text { knowledge increased (pretest vs posttest) }\end{array}$ \\
\hline $\begin{array}{l}\text { O'Connor } \\
\text { et al, },^{32} 2016\end{array}$ & $\begin{array}{l}\text { Radiology clerkship } \\
\text { (M3 and M4) }\end{array}$ & $\begin{array}{l}\text { Web-based } \\
\text { tutorials }\end{array}$ & $\begin{array}{l}\text { Interactive } \\
\text { workshops }\end{array}$ & $\begin{array}{l}\text { Control } \\
\text { cohort (LB) }\end{array}$ & $\begin{array}{l}\text { Increased enjoyment, task value, and reduced boredom } \\
\text { (student surveys) and better student performance; } \\
\text { instructors noted preference for FC approach }\end{array}$ \\
\hline $\begin{array}{l}\text { Duque } \\
\text { et al, },^{33} 2013\end{array}$ & $\begin{array}{l}\text { Geriatric medicine } \\
(\mathrm{M} 3 \text { and } M 4)\end{array}$ & $\begin{array}{l}\text { e-Learning } \\
\text { modules, video } \\
\text { game }\end{array}$ & $\begin{array}{l}\text { Weekly case } \\
\text { conference with } \\
\text { interprofessional } \\
\text { team }\end{array}$ & None & $\begin{array}{l}\text { Approach led to positive attitudes regarding geriatric } \\
\text { medicine; knowledge increased (assessed via pretests } \\
\text { vs posttests) as a function of modules }\end{array}$ \\
\hline $\begin{array}{l}\text { Ingrassia } \\
\text { et } \mathrm{al}^{34} 2014\end{array}$ & $\begin{array}{l}\text { Blended learning } \\
\text { approach to disaster } \\
\text { medicine (M4-M6) }\end{array}$ & $\begin{array}{l}\text { e-Learning } \\
\text { modules }\end{array}$ & $\begin{array}{l}\text { Workshops } \\
\text { including PBL and } \\
\text { simulation activities }\end{array}$ & None & $\begin{array}{l}\text { Student surveys characterized generally strong } \\
\text { perceptions; knowledge increased (pretest vs posttest) }\end{array}$ \\
\hline $\begin{array}{l}\text { Boysen- } \\
\text { Osborn } \\
\text { et } a{ }^{3,}, 2016\end{array}$ & $\begin{array}{l}\text { Advanced cardiac } \\
\text { life support }\end{array}$ & Podcasts & TBL activities & $\begin{array}{l}\text { Previous } \\
\text { cohorts (LB) }\end{array}$ & Small improvements in examination results \\
\hline $\begin{array}{l}\text { Robinson, }{ }^{36} \\
2016\end{array}$ & $\begin{array}{l}\text { Medicine as business } \\
\text { elective (M4) }\end{array}$ & Online videos & $\begin{array}{l}\text { Small group } \\
\text { discussion-based } \\
\text { activities }\end{array}$ & $\begin{array}{l}\text { Subsequent } \\
\text { cohort with } \\
\text { MOOC } \\
\text { approach }\end{array}$ & $\begin{array}{l}\text { Student evaluations were similar for } \mathrm{MOOC} \text { and FC } \\
\text { approaches }\end{array}$ \\
\hline
\end{tabular}

Note: aStudent perception data related to this innovation was derived from personal communication (Yiu et al, 20I6).

Abbreviations: FC, flipped classroom; TBL, team-based learning; CBL, case-based learning; MI, first-year medical students; M2, second-year medical students; M3, third year medical students; M4, fourth-year medical students; M5, fifth-year medical students; M6, sixth-year medical students; EBM, evidence-based medicine; LB, lecture-based or teacher-centered approach; MOOC, massive open online course; NBME, National Board of Medical Examiners; OSCEs, Objective Structured Clinical Examinations. 
to temporal considerations related to their applications of the FC in clinical medical student education, as was sometimes the case for preclinical FCs.

\section{Pre-class preparation activities in flipped undergraduate medical education}

Pre-session electronic resources for medical student self-directed content orientation often took the format of online content videos that typically featured short lectures ${ }^{11,14,17,18,20-22,28,29,33}$ or slide presentations with accompanying narration (Tables 2 and 3). ${ }^{14,19,25,27}$ Other resources frequently provided to medical students included audiobased resources (eg, podcasts), ${ }^{15,30,35}$ e-learning tools, ${ }^{13,24,32}$ and educational games. ${ }^{31,33}$ Finally, text-based resources (eg, e-books and research articles) were typically provided as supplemental (ie, additional reading) preparatory resources. ${ }^{12,13,17,26,34}$ In a case where multiple resources were provided for pre-class preparation, students reported accessing video-based resources more frequently than other tools. ${ }^{17}$ In some instances, formative learning (eg, short quizzes) was provided as part of the preparatory material, so that students could self-assess their understanding of the presented content. ${ }^{11,17,20,28,29,31}$ Although in most cases educators developed their own site-specific video resources, there was at least one instance of utilization of open access videos. ${ }^{22}$

\section{In-class flipped medical student activities}

The most common modalities used to promote student engagement and active learning in the classroom component were clinical case-based small group activities, , $3,17,22,26-29,31^{2}$ which in many cases could be considered to be modified versions of conventional case-based learning (CBL; Tables 2 and 3). Other validated active learning methods that were modified and applied in FC face-to-face sessions included problem-based learning (PBL) ${ }^{25,34}$ and team-based learning (TBL). ${ }^{15,18,31,35}$ The authors did not, in many articles, explicitly describe how their methodology precisely applied each specific element of conventional CBL, PBL, and TBL, so it is likely that modified versions of the conventional approaches were used.

Other in-class active learning sessions included student-driven workshops ${ }^{23,32,34}$ or discussion-based activities, ${ }^{11,14,17,22,36}$ which typically took place in small group settings, though one initiative described a large group discussion that applied the "think/pair/share" active learning approach. ${ }^{14}$ One clinical FC initiative incorporated weekly conference discussions that had students engage with their interprofessional teammates. ${ }^{33}$ In some cases when formative quizzes were incorporated into the pre-session preparation activities, formal class time was dedicated to review of the formative exercises with faculty. ${ }^{28,29}$ In other instances, low-stakes quizzes were incorporated into the face-to-face sessions as part of assessing student preparedness, a hallmark element of TBL. ${ }^{15,18,31,35}$ Invariably, across the different teaching modalities used, educators present in face-to-face teaching sessions served as facilitators, helping (at most) to guide the inquiry and application activities by medical students..$^{15,32}$

\section{Medical student perceptions: non- knowledge-based benefits of the FC}

Almost universally, FCs in undergraduate medical education have been well received by medical students in both preclinical (Table 2) and clinical (Table 3) applications, generating strong positive perceptions on student evaluation surveys. ${ }^{11,12,16-20,22,24,26-30,32,34}$ Medical students generally express high degrees of satisfaction with online videos specifically designed for pre-class preparation in FC initiatives. Students have reported particular appreciation for concise, engaging, multimodal learning resources that can be accessed by students at any time and as often as they desire. . $^{17,18,28,29,31}$ One recent study of a FC application in emergency medicine qualitatively compared feedback between a group of students who received a live lecture on trauma management and a group that instead used open access online resources (https:// flippedemclassroom.wordpress.com/2014/04/14/approachto-trauma-by-stella-yiu/) for their pre-session preparation (Yiu, O’Brien, Day, Frank, unpublished data). Interestingly, the groups exhibited strong preferences for the teaching modality to which they were exposed. Furthermore, based on qualitative data, it was suggested that students may perceive a blended curriculum (incorporating both lecture-based and FC modalities) to be best suited to their learning preferences.

The incorporation of active learning strategies in face-toface sessions in FC initiatives has also been highly regarded by medical students. Students perceive that the active learning sessions increase their motivation to learn ${ }^{12}$ and enhance their level of engagement, investment, and interest in the subject matter. ${ }^{15,16,22,28,30}$ Relatedly, when explicitly queried, medical students typically indicate a strong preference for the interactive active learning environments in the $\mathrm{FC}$ relative to traditional lecture-based instruction. ${ }^{19,22,31,32,36}$

In general, the aspects of FC applications that are especially valued by medical students include the incorporation of self-directed learning, ${ }^{23,28}$ active learning, ${ }^{15,26,27}$ and exercises that facilitate peer interaction. ${ }^{15,22,30}$ One student survey-based finding indicated an appreciation for the positive impact that 
the FC application (one that included pre-session formative quizzes and in-session review of those quizzes) had on student accountability. ${ }^{29}$ As such, medical students have found such approaches to be valuable and highly satisfying educational activities. ${ }^{17-20,32}$ In some cases, the FC yielded positive effects on student attitudes toward the presented subject matter. For example, an increase in self-reported student interest in surgically oriented careers was reported in response to a FC in surgery clerkship. ${ }^{28}$ In addition, the incorporation of flipped elements in a blended learning approach to geriatric medicine improved student attitudes toward the subject. ${ }^{33}$

\section{Medical student perceptions: does the FC method measurably improve knowledge?}

Several studies have indicated that medical students perceive that the FC approach benefits their knowledge and their learning. ${ }^{12,19,22,23,30}$ There may be limited evidence in support of these notions. Although several FC initiatives in undergraduate medical education have indicated benefits to learning using pretest vs posttest scores, ${ }^{26,30-34}$ only a few of these studies demonstrated an improvement relative to the pretest vs posttest improvements achieved via other modalities (Table 3). ${ }^{31,32}$ In contrast, other findings have indicated that the FC does not significantly, consistently, or meaningfully improve student performance on examinations (Tables 2 and 3). ${ }^{14,17,19,23-25,27,35}$ For example, FC approaches to EBM did not increase scores on the Berlin objective assessment of EBM competencies (relative to control groups receiving traditional instruction), despite medical students perceiving an increase in skills due to the intervention. ${ }^{23,24}$ Moreover, a FC application in an obstetrics-gynecology clerkship generally improved student performance (relative to students receiving traditional teaching) in several subject areas but inexplicably led to decreased performance in items related to gynecology. Two of the included studies determined that FC applications in clerkships (surgery and obstetrics and gynecology) did not alter student performance (relative to historical cohorts with traditional instruction) on the relevant items on the National Board of Medical Examiners (NBME) assessments. ${ }^{27,28}$ Finally, student-centered learners in a modified FC/TBL approach to anatomy laboratory exercises did not perform as well as those exposed to a more faculty-centered approach. ${ }^{15}$

The question arises as to why discrepancies exist regarding the benefits (if any) of the FC on knowledge gained, as determined by scores on assessment tools. The discrepancies that exist may be, at least in part, due to the nature of the assessment tools used. When a FC approach was applied to a preclinical anatomy course, medical student performance (relative to that of a prior cohort exposed to a more traditional, didactic approach) did not improve on items that assessed knowledge (lower cognitive skills). ${ }^{14}$ However, when isolating items that assessed analysis, students exposed to the FC outperformed those exposed to conventional teaching.

\section{Medical student perceptions: caveats of the FC}

Although the overwhelming response from medical students exposed to the $\mathrm{FC}$ approach is positive, there are concerns that have emerged in the literature regarding several aspects of inverted learning. Regarding the pre-session phase, medical students have expressed concern over the time-intensiveness of some of the material provided for content orientation. ${ }^{12,17,31}$ In addition, qualitative feedback from students utilizing FC online resources in emergency medicine noted that online learning is limited in terms of ability to access and ask questions of faculty and interact with classmates (Yiu, O'Brien, Day, Frank, unpublished data). In addition, certain students have expressed that pre-class learning tools need to be appropriately aligned (in terms of detail, difficulty, and relevance) with subsequent in-class learning objectives. For example, in one study, some students viewed their preparatory material as being too basic relative to the content covered in subsequent classroom sessions. ${ }^{22}$ In contrast, in other settings, students opined that some subject matter was overly complex to be delivered strictly with self-directed learning tools and without faculty support. ${ }^{13,19}$

Medical students are generally satisfied with the in-class active learning component of the FC. However, a minority of students have suggested that certain aspects of the active learning components of their flipped medical education classrooms could be improved. In one FC application, student commentary indicated that some inefficiency in the active learning component was attributed to face-to-face classroom time being dedicated to basic material already well covered by pre-class resources. ${ }^{30}$ Similarly, some active learning activities (TBL, CBL, and facilitated workshops) were perceived by a small proportion of students to be inefficient in terms of students achieving learning objectives..$^{15,22,23,31}$ These inefficiencies in active learning settings were attributed by student commentary to be, at least in part, due to inadequate preparation of students. ${ }^{15,22,23}$ In addition, some small group discussions could benefit from increased structure (ie, keeping students on task) and facilitating contributions from students who tend to be less vocal, while tempering the contributions of students who tend to dominate. ${ }^{22}$ In some instances where progression through learning objectives 
may be less than optimal, students suggested that learning would be improved with increased direction and support from faculty. ${ }^{15,23}$ However, faculty who are overly didactic in active learning contexts risk disengaging students and may negate the proven benefits of student-centered strategies. ${ }^{15}$ Finally, one study noted that a massive open online course (a course without a face-to-face, active learning component) and a FC approach to medicine as a business both generated similar, positive student responses. This finding advances the notion that student-centered, face-to-face active learning environments could be replaced without detriment to student satisfaction, in some circumstances. ${ }^{36}$

\section{Evidence corroborating medical student perceptions}

Although the majority of studies characterizing increased medical student interest, engagement, and value for education delivered in FCs have relied heavily on surveys of student perceptions, there is some objective evidence that corroborates student self-reported notions. For example, when a first-year biochemistry course was flipped from the traditional lecturebased model to one that incorporated pre-session short videos and in-session clinical case discussions, student attendance (a surrogate for student enthusiasm) increased from 30\% to $80 \% .{ }^{11}$ Another study demonstrated high video access counts (>95\%) and attendance (>93\%) across various FC modules in surgery clerkship as evidence of student engagement. ${ }^{28}$ Finally, one evaluation of a FC application in emergency medicine clerkship included observation and individual ratings of students by their small group facilitators. ${ }^{22}$ Facilitators rated students highly in terms of preparedness, participation levels, and knowledge based on observed interactions in the case-based discussions, indicative of high levels of student engagement and investment in this educational approach.

Although the studies chosen for this review were based on medical student perceptions and outcomes regarding the FC, two of these studies also characterized the views of faculty participants. In one initiative introducing POCUS to first-year medical students, faculty surveyed prior to the course indicated a strong sense of value regarding the intervention, and this vantage did not change when surveyed postcourse. ${ }^{20}$ Another study characterized the view of four instructors in a multi-institutional (three sites) application of the FC to radiology clerkship. Although all instructors saw positive aspects to traditional didactic instruction, they tended to be in strong agreement with the notion that the FC environment promoted student learning. Furthermore, the instructors indicated a preference for FC teaching relative to didactic lecturing, ${ }^{32}$ consistent with the views of medical students. Similarly, instructors noted that variability in (or lack of) student preparedness and willingness to participate can negatively impact the educational value of interactive learning sessions, mirroring student perceptions.

\section{Discussion}

Although the flipped or inverted classroom has been in existence for many years, ${ }^{1,7,9,37}$ this modality is relatively new to undergraduate medical education. The first documented use of the FC in teaching medical students was only recently published in 2012.11 Many medical educators have since introduced elements of the FC to their teaching. In this review of disseminated applications of FC methodology to undergraduate medical education, it is clear that there are many aspects that are particularly well received by medical students. However, there remain certain aspects of the FC that could be improved from the medical student perspective, and these medical student perceptions are worth considering in future applications of the FC in medical education.

Medical students have demonstrated general appreciation for specific features of pre-class preparatory material (conciseness, relevance, accessibility, and multimodality) that could have been predicted based on technology-assisted learning theories ${ }^{38}$ and established applications of these theories to FC resources. ${ }^{21,39}$ However, there was evidence in the reviewed studies that, in some cases, students perceive that there needs to be improved congruency between the complexity and detail of pre-session preparatory material and those of the corresponding active learning session. This may explain why the use of open access FCs was limited to only one of the reviewed studies. ${ }^{22}$ Educators may feel the need to invest time and effort into producing online content specific to their local curricula, as students may find that specific content optimal. Although faculty feedback regarding the design of FC resources was not captured in any of the reviewed studies, it has been suggested that the upfront time investments required to produce appropriate resources could be perceived as a barrier to adopting the flipped teaching approach. ${ }^{39}$

The active learning environments of face-to-face sessions have been highly regarded by medical students. Specifically, these components have been perceived to be enjoyable, self-directed, interactive, and engaging because of the incorporation of established active learning strategies grounded in adult educational theory. As a result, student reception of $\mathrm{CBL}, \mathrm{TBL}$, and $\mathrm{PBL}$ applications was largely positive in all cases. The student concerns raised largely 
focused on perceived inefficiency in learning during these student-centered approaches. Some of this inefficiency was acknowledged by students to be the result of inadequate preclass preparation by learners, and it is well accepted that these learning strategies are dependent on student preparedness. Dedicating a small amount of in-class time to reviewing of pre-class formative quizzes, as was done in $\mathrm{FC}$ applications to surgery clerkship, ${ }^{28,29}$ may help improve student accountability. Alternatively, individual and group preparedness could be formally assessed using low-stakes quizzes at the beginning of in-class sessions, which is done in conventional TBL and is proven to enhance student accountability. ${ }^{40}$

Other aspects that could detract from the student-centered approaches could be effectively dealt with by appropriate direction by small group teachers. In our local application of modified TBL to the anatomy laboratory, some student groups perceived a lack of direction and support related to achieving learning objectives. Furthermore, others felt that their group activities were not conducive to active learning as their teachers tended to dominate discussions. ${ }^{15}$ It is important to note that, in the in-class component of the FC, the faculty's role should be that of a facilitator. Facilitators should be able to support and provide some structure for students as they drive progress through learning objectives (guided inquiry). ${ }^{18,32}$ Although faculty may embrace and enjoy their roles as facilitators in $\mathrm{FCs},{ }^{32}$ this teaching role is not necessarily easy. This is particularly the case for many faculty who may have been influenced by teacher-centered learning in their own education, and who themselves have spent most of their careers as teacher-centered educators. In our local case, we addressed the variability in student experience in the following ways: 1) we adapted and increased our training of our faculty in small group facilitation skills; 2) to enhance student self-reliance during laboratory sessions, we created resources to facilitate student-driven learning in laboratory (itemized checklists) and enhance student preparation prior to laboratory (formative quizzes); and 3) we reduced the faculty to student ratio (from 1 facilitator/group to 1 facilitator/2 groups) to allow for more time for students to drive their own learning (and to prevent facilitators from reverting to lecture-based teaching). The resulting changes have resolved most of the issues concerning the variable active learning environments between groups (Ramnanan, unpublished data).

Although the FC is widely considered in the reviewed literature to be beneficial to medical student learning, this notion is limited by the fact that the supporting evidence has largely been culled from surveys of student perceptions and is therefore subject to inherent biases (eg, selection biases associated with surveys). The question persists as to whether these strategies actually enhanced learning. The difficulty and inconsistency of objectively demonstrating an academic learning benefit in the literature may be, at least in part, due to the assessment tools being used. For example, enhanced student performance resulting from a FC application in anatomy was not observed, when evaluating items that assessed knowledge or application (lower cognitive levels in Bloom's taxonomy). ${ }^{14}$ However, students exposed to the flipped model did have stronger performance on items that assessed higher levels of cognition. This brings to light the need for assessment tools to evolve, mirror, and appropriately assess the changes that have occurred in teaching. It is conceivable that the FC may not measurably improve academic performance on undergraduate multiple choice examinations or even NBME examinations. ${ }^{27,28}$ However, regardless of enhancement of examination scores related to content delivered in the flipped approach, student-centered and active learning principles applied in the FC should enhance and improve lifelong learning skills, which are desirable competencies in medical student trainees. Further study needs to confirm whether long-term lifelong learning competencies are actually enhanced by FC innovations in medical school.

It must be noted that this review focused on FC applications specifically in undergraduate medical education, since there is great potential for this innovation to replace the teacher-centered strategies that are currently widely used during this period of medical training, thereby addressing formal accreditation standards driving medical school curricular development. In postgraduate medical education, application of FC methodology could be considered a current educational trend as well, ${ }^{41,42}$ with implementation of the approach described in several recent studies involving residents. ${ }^{43,44}$ Future studies will determine the effectiveness of flipped approaches for this level of medical learner.

\section{Conclusion}

To date, the early student response to FC applications in undergraduate medical education is largely positive. The studies characterized thus far have demonstrated strong student satisfaction with pre-class learning resources that have been designed based on sound technology-assisted learning theory. Moreover, students generally express strong satisfaction with learner-centered approaches in classes and preference for active learning modalities over traditional lecture-based learning. Although typically only few students express concerns (eg, lack of student preparedness and 
variable learning environments across small groups) with the face-to-face components of the FC, these concerns may be addressed by introducing elements that reinforce student accountability and student-centeredness in activities. This includes appropriate training for facilitators who teach the small group components in FCs. Although there may not be convincing evidence indicating that the FC enhances learning (as assessed by student examination performance), this may reflect the nature of the assessment tools used. Regardless of benefits on academic examinations, the FC does not appear to hinder learning and can help develop valuable lifelong learning skills in undergraduate medical students. For these reasons, more medical schools and medical educators are likely to rely on the FC going forward.

\section{Disclosure}

The authors report no conflicts of interest in this work.

\section{References}

1. Mazur E. Education. Farewell, lecture? Science. 2009;323(5910):50-51.

2. Taylor DC, Hamdy H. Adult learning theories: implications for learning and teaching in medical education: AMEE Guide No. 83. Med Teach. 2013;35(11):e1561-e1572.

3. Graffam B. Active learning in medical education: strategies for beginning implementation. Med Teach. 2007;29(1):38-42.

4. Committee on the Accreditation of Canadian Medical Schools. CACMS Standards and Elements: Standards for Accreditation of Medical Education Programs Leading to the M.D. Degree. Ottawa, Canada; 2015.

5. Brame CJ. Flipping the classroom. Vanderbilt University Center for Teaching; 2013. Available from: https://cft.vanderbilt.edu/guides-subpages/flipping-the-classroom/. Accessed September 24, 2016.

6. Pierce R, Fox J. Vodcasts and active-learning exercises in a "flipped classroom" model of a renal pharmacotherapy module. Am J Pharm Educ. 2012;76(10):196.

7. Bishop JL, Verleger MA. The flipped classroom: a survey of the research. Paper presented at: ASEE National Conference Proceedings; 2013; Atlanta, GA.

8. McLaughlin JE, Roth MT, Glatt DM, et al. The flipped classroom: a course redesign to foster learning and engagement in a health professions school. Acad Med. 2014;89(2):236-243.

9. O'Flaherty J, Phillips C. The use of flipped classrooms in higher education: a scoping review. Int High Educ. 2015;25:85-95.

10. Arksey H, O’Malley L. Scoping studies: towards a methodological framework. Int J Soc Res Met. 2005;8(1):19-32.

11. Prober CG, Heath C. Lecture halls without lectures - a proposal for medical education. N Engl J Med. 2012;366(18):1657-1659.

12. de Fátima Wardenski R, de Espíndola MB, Struchiner M, Giannella TR. Blended learning in biochemistry education: analysis of medical students' perceptions. Biochem Mol Biol Educ. 2012;40(4):222-228.

13. Veeramani R, Madhugiri VS, Chand P. Perception of MBBS students to "flipped class room" approach in neuroanatomy module. Anat Cell Biol. 2015;48(2):138-143.

14. Morton DA, Colbert-Getz JM. Measuring the impact of the flipped anatomy classroom: the importance of categorizing an assessment by Bloom's taxonomy. Anat Sci Educ. In press 2016.

15. Whelan A, Leddy JJ, Mindra S, Matthew Hughes JD, El-Bialy S, Ramnanan CJ. Student perceptions of independent versus facilitated small group learning approaches to compressed medical anatomy education. Anat Sci Educ. 2016;9(1):40-51.
16. Grossman E, Grosseman S, Azevedo GD, Figueiro-Filho EA, McKinley D. Flipped classroom on humanities: medicine, narrative and art. Med Educ. 2015;49(11):1142.

17. Evans KH, Thompson AC, O'Brien C, et al. An innovative blended preclinical curriculum in clinical epidemiology and biostatistics: impact on student satisfaction and performance. Acad Med. 2016;91(5): 696-700.

18. Sharma N, Lau CS, Doherty I, Harbutt D. How we flipped the medical classroom. Med Teach. 2015;37(4):327-330.

19. Sajid MR, Laheji AF, Abothenain F, Salam Y, AlJayar D, Obeidat A. Can blended learning and the flipped classroom improve student learning and satisfaction in Saudi Arabia? Int J Med Educ. 2016;7: 281-285.

20. Nelson BP, Hojsak J, Dei Rossi E, Karani R, Narula J. Seeing is believing: evaluating a point-of-care ultrasound curriculum for 1st-year medical students. Teach Learn Med. 2016:1-8.

21. Patwari R, Yiu S. Toolkit to flip your classroom. Paper presented at: International Conference for Residency Education. Invited Workshop; 2014; Toronto, Canada.

22. Lew EK. Creating a contemporary clerkship curriculum: the flipped classroom model in emergency medicine. Int J Emerg Med. 2016;9(1):25.

23. Ilic D, Hart W, Fiddes P, Misso M, Villanueva E. Adopting a blended learning approach to teaching evidence based medicine: a mixed methods study. BMC Med Educ. 2013;13:169.

24. Ilic D, Nordin RB, Glasziou P, Tilson JK, Villanueva E. A randomised controlled trial of a blended learning education intervention for teaching evidence-based medicine. BMC Med Educ. 2015;15:39.

25. Gillispie V. Using the flipped classroom to bridge the gap to generation Y. Ochsner J. 2016;16(1):32-36.

26. Morgan H, Marzano D, Lanham M, Stein T, Curran D, Hammoud M. Preparing medical students for obstetrics and gynecology milestone level one: a description of a pilot curriculum. Med Educ Online. 2014;19:25746.

27. Morgan H, McLean K, Chapman C, Fitzgerald J, Yousuf A, Hammoud M. The flipped classroom for medical students. Clin Teach. 2015;12(3):155-160.

28. Liebert CA, Lin DT, Mazer LM, Bereknyei S, Lau JN. Effectiveness of the surgery core clerkship flipped classroom: a prospective cohort trial. Am J Surg. 2016;211(2):451-457.e1.

29. Liebert CA, Mazer L, Bereknyei Merrell S, Lin DT, Lau JN. Student perceptions of a simulation-based flipped classroom for the surgery clerkship: a mixed-methods study. Surgery. 2016;160(3):591-598.

30. Bosner S, Pickert J, Stibane T. Teaching differential diagnosis in primary care using an inverted classroom approach: student satisfaction and gain in skills and knowledge. BMC Med Educ. 2015;15:63.

31. Belfi LM, Bartolotta RJ, Giambrone AE, Davi C, Min RJ. "Flipping" the introductory clerkship in radiology: impact on medical student performance and perceptions. Acad Radiol. 2015;22(6):794-801.

32. O'Connor EE, Fried J, McNulty N, et al. Flipping radiology education right side up. Acad Radiol. 2016;23(7):810-822.

33. Duque G, Demontiero O, Whereat S, et al. Evaluation of a blended learning model in geriatric medicine: a successful learning experience for medical students. Australas J Ageing. 2013;32(2):103-109.

34. Ingrassia PL, Ragazzoni L, Tengattini M, Carenzo L, Della Corte F. Nationwide program of education for undergraduates in the field of disaster medicine: development of a core curriculum centered on blended learning and simulation tools. Prehosp Disaster Med. 2014;29(5):508-515.

35. Boysen-Osborn M, Anderson CL, Navarro R, et al. Flipping the advanced cardiac life support classroom with team-based learning: comparison of cognitive testing performance for medical students at the University of California, Irvine, United States. J Educ Eval Health Prof. 2016;13:11.

36. Robinson R. Delivering a medical school elective with massive open online course (MOOC) technology. Peer J. 2016;4:e2343. 
37. Lage MJ, Platt GJ, Treglia M. Inverting the classroom: a gateway to creating an inclusive learning environment. J Econ Educ. 2000;31(1):30-43.

38. Masters K, Ellaway R. e-Learning in medical education Guide 32 Part 2: technology, management and design. Med Teach. 2008;30(5): 474-489.

39. Moffett J. Twelve tips for "flipping" the classroom. Med Teach. 2015; 37(4):331-336.

40. Michaelsen LK, Sweet M. The essential elements of team-based learning. N Dir Teach Learn. 2008;2008(116):7-27.

41. Mayorga E, Golnik K, Palis G. One-year progress in ophthalmic education: annual review. Asia Pac J Ophthalmol (Phila). 2015;4(6):388-398.
42. Turco MG, Baron RB. Observations on the 2016 world congress on continuing professional development: advancing learning and care in the health professions. J Contin Educ Health Prof. 2016;36(Suppl 1): S4-S7.

43. Rose E, Claudius I, Tabatabai R, Kearl L, Behar S, Jhun P. The flipped classroom in emergency medicine using online videos with interpolated questions. J Emerg Med. 2016;51(3):284-291.

44. Tainter CR, Wong NL, Cudemus-Deseda GA, Bittner EA. The "flipped classroom" model for teaching in the intensive care unit: rationale, practical considerations, and an example of successful implementation. J Intensive Care Med. 2016.

\section{Publish your work in this journal}

Advances in Medical Education and Practice is an international, peerreviewed, open access journal that aims to present and publish research on Medical Education covering medical, dental, nursing and allied health care professional education. The journal covers undergraduate education, postgraduate training and continuing medical education including emerging trends and innovative models linking education, research, and health care services. The manuscript management system is completely online and includes a very quick and fair peer-review system. Visit http://www.dovepress.com/testimonials.php to read real quotes from published authors.

Submit your manuscript here: http://www.dovepress.com/advances-in-medical-education-and-practice-journal 